\title{
LINEAR DYNAMICS IN REPRODUCING KERNEL HILBERT SPACES
}

\author{
ANEESH MUNDAYADAN AND JAYDEB SARKAR \\ Dedicated to Professor Kalyan Bidhan Sinha on the occasion of his 75th birthday
}

\begin{abstract}
Complementing earlier results on dynamics of unilateral weighted shifts, we obtain a sufficient (but not necessary, with supporting examples) condition for hypercyclicity, mixing and chaos for $M_{z}^{*}$, the adjoint of $M_{z}$, on vector-valued analytic reproducing kernel Hilbert spaces $\mathcal{H}$ in terms of the derivatives of kernel functions on the open unit disc $\mathbb{D}$ in $\mathbb{C}$. Here $M_{z}$ denotes the multiplication operator by the coordinate function $z$, that is

$$
\left(M_{z} f\right)(w)=w f(w),
$$

for all $f \in \mathcal{H}$ and $w \in \mathbb{D}$. We analyze the special case of quasi-scalar reproducing kernel Hilbert spaces. We also present a complete characterization of hypercyclicity of $M_{z}^{*}$ on tridiagonal reproducing kernel Hilbert spaces and some special classes of vector-valued analytic reproducing kernel Hilbert spaces.
\end{abstract}

\section{INTRODUCTION}

Motivated by challenges in the dynamics of bounded linear operators on Banach spaces, in this paper, we initiate the study of dynamics of adjoints of the multiplication operators on analytic reproducing kernel Hilbert spaces. Here we bring together two sets of ideas the Hypercyclicity Criterion and analytic reproducing kernels on the open unit disc $\mathbb{D}$ in $\mathbb{C}$.

More specifically, motivated by Salas' work [27] on the characterization of hypercyclicity of backward weighted shift operators on $l^{p}$ spaces, $1 \leq p<\infty$, we propose a general question on hypercyclicity of the adjoints of the multiplication operators on analytic reproducing kernel Hilbert spaces. In Theorems 4.1 and 4.2, we present sufficient conditions for testing hypercyclicity, topological mixing and chaoticity of adjoints of the multiplication operators on reproducing kernel Hilbert spaces in terms of derivatives of analytic kernel functions.

In the special case of scalar-valued analytic kernel functions, our main theorem on hypercyclicity states the following (see Theorem 5.1): Let $k: \mathbb{D} \times \mathbb{D} \rightarrow \mathbb{C}$ be an analytic kernel function and let $\mathcal{H}(k)$ denote the reproducing kernel Hilbert space corresponding to $k$ (see Section 3 for definitions). Suppose that the multiplication operator $M_{z}$ on $\mathcal{H}(k)$ is bounded. Then $M_{z}^{*}$ on $\mathcal{H}(k)$ is hypercyclic if

$$
\liminf _{n}\left(\frac{1}{(n !)^{2}} \frac{\partial^{2 n} k}{\partial z^{n} \partial \bar{w}^{n}}(0,0)\right)=0 .
$$

2010 Mathematics Subject Classification. Primary 47A16, 46E22, 32K05, 47B32; Secondary 47B37, 37 A99.

Key words and phrases. hypercyclicity, chaos, mixing, reproducing kernel Hilbert spaces, multiplication operator, linear dynamics. 
In the Hilbert space setting, this result unifies previous work on sufficient conditions for hypercyclicity of weighted shift operators (see Gethner and Shapiro [17], Kitai [21], Rolewicz [26], and Salas [27]).

The sufficient condition for hypercyclicity in Theorem 4.1 is not necessary, in general (see the example in Subsection 5.3).

For the context of hypercyclicity of translation operators on reproducing kernel Hilbert spaces of entire functions, we refer the reader to Baranov [4], Chan and Shapiro [13], Garcia, Hernandez-Medina and Portal [16] and Godefroy and Shapiro [18]. Also see Bonet [10].

After a preliminary section devoted to fixing the notations and recalling the basic facts about dynamics of bounded linear operators, in Section 3, we describe the necessary background of reproducing kernel Hilbert spaces. Some of our results are less standard. Moreover, it is worth pointing out that the "backward shift" behavior of the adjoints of multiplication operators on analytic reproducing kernel Hilbert spaces in Theorem 3.3 is of independent interest.

Section 4 contains the main results on dynamics of $M_{z}^{*}$ on analytic reproducing kernel Hilbert spaces. In Section 5, we provide an explicit example of an analytic reproducing kernel space on which $M_{z}^{*}$ is hypercyclic but does not satisfy the sufficient condition for hypercyclicity in Theorem 4.1. The final section deals with the converse of Theorem 4.1 for tridiagonal reproducing kernel Hilbert spaces and a class of vector valued analytic reproducing kernel Hilbert spaces.

\section{PRELiminaries}

In this section we fix some notation and recall definitions and facts on dynamics of bounded linear operators. For more details concerning linear dynamics, we refer the reader to the monographs by F. Bayart and E. Matheron [6] and K.G. Grosse-Erdmann and A. Peris [20].

Let $X$ be a Banach space and let $\mathcal{B}(X)$ be the set of all bounded linear operators on $X$. Let $T$ be a bounded linear operator on $X$. We say that $T$ is:

(i) hypercyclic if there exists $x \in X$ such that

$$
\left\{x, T x, T^{2} x, \ldots\right\}
$$

is dense in $X$,

(ii) chaotic if $T$ is hypercyclic and it has a dense set of periodic points (a vector $y$ in $X$ is called periodic for $T$ if there exists $k \in \mathbb{N}$ such that $T^{k} y=y$ ), and

(iii) topologically transitive, if

$$
\bigcup_{m=0}^{\infty} T^{-m}(U),
$$

is dense in $X$ for every non-empty open set $U$ in $X$.

From the point of view of hypercyclic operators, in this paper, Banach spaces are always assumed to be separable.

A well known theorem of Birkhoff, known as Birkhoff's transitivity theorem, states that (cf. [6]): $T$ in $\mathcal{B}(X)$ is hypercyclic if and only if $T$ is topologically transitive.

Another important notion in dynamical systems is topological mixing: $T$ in $\mathcal{B}(X)$ is said to be topologically mixing if, given any two non-empty open subsets $U$ and $V$ of $X$, 
there exists $N$ in $\mathbb{N}$ such that

$$
T^{-j}(U) \cap V \neq \varnothing,
$$

for all $j \geq N$.

Classical examples of hypercyclic operators are translation operators [9], differential operators [23] and commutators of translation operators [18]. The first example of a hypercyclic backward weighted shift was produced by Rolewicz [26]: for each $1 \leq p<\infty$ and $|\alpha|>1$, the backward weighted shift $\alpha B$ is hypercyclic on $l^{p}$, where $B$ on $l^{p}$ is the unweighted backward shift:

$$
B\left(\left\{c_{0}, c_{1}, c_{2}, \ldots\right\}\right)=\left\{c_{1}, c_{2}, c_{3} \ldots\right\},
$$

for all $\left\{c_{0}, c_{1}, c_{2} \ldots\right\} \in l^{p}$ (see also Kitai [21] and Gethner and Shapiro [17]). Salas [27] provides a further improvement by characterizing hypercyclicity of backward weighted shifts: Let $\lambda=\left\{\lambda_{n}\right\}_{n \geq 1}$ be a bounded sequence of positive real numbers. Then the backward weighted shift $B_{\lambda}$ on $l^{p}, 1 \leq p<\infty$, defined by

$$
B_{\lambda}\left(\left\{c_{0}, c_{1}, c_{2}, \ldots\right\}\right)=\left\{\lambda_{1} c_{1}, \lambda_{2} c_{2}, \lambda_{3} c_{3}, \ldots\right\}
$$

for all $\left\{c_{0}, c_{1}, c_{2}, \ldots\right\} \in l^{p}$, is hypercyclic if and only if

$$
\limsup _{n}\left\{\lambda_{1} \lambda_{2} \cdots \lambda_{n}\right\}=\infty .
$$

For our purposes, we interpret the $p=2$ case from a function Hilbert space [28] point of view: Given a sequence of positive real numbers $\beta=\left\{\beta_{n}\right\}_{n \geq 0}$, we write $H^{2}(\beta)$ for the Hilbert space of all formal power series

$$
f(z)=\sum_{n \geq 0} a_{n} z^{n}
$$

such that

$$
\|f\|_{H^{2}(\beta)}^{2}:=\sum_{n \geq 0} \frac{\left|a_{n}\right|^{2}}{\beta_{n}^{2}}<\infty .
$$

Clearly, the set of functions $\left\{\beta_{n} z^{n}\right\}$ forms an orthonormal basis in $H^{2}(\beta)$. If, in addition, we assume that

$$
\limsup _{n} \frac{\beta_{n+1}}{\beta_{n}} \leq 1,
$$

then $H^{2}(\beta) \subseteq \mathcal{O}(\mathbb{D})$ (cf. Shields [28]), the space of all analytic functions on $\mathbb{D}$. It also follows that the multiplication operator $M_{z}$ on $H^{2}(\beta)$ defined by

$$
\left(M_{z} f\right)(w)=w f(w)
$$

for all $f \in H^{2}(\beta)$ and $w \in \mathbb{D}$, is bounded if and only if

$$
\sup _{n} \frac{\beta_{n}}{\beta_{n+1}}<\infty
$$

In this case, Salas' classification result can be interpreted as follows: $M_{z}^{*}$ is hypercyclic on $H^{2}(\beta)$ if and only if

$$
\liminf _{n} \beta_{n}=0 .
$$

Moreover, the Costakis-Sambarino theorem [15] states that: $M_{z}^{*}$ is mixing if and only if

$$
\lim _{n} \beta_{n}=0 .
$$


The well-known and useful sufficient conditions for hypercyclicity of operators on Banach spaces states the following, (see Kitai [21, Gethner and Shapiro [17, Godefroy and Shapiro [18], and Bes and Peris [8]): Let $X$ be a Banach space, $D$ be a dense set in $X$, and let $T \in \mathcal{B}(X)$. If there exist a strictly increasing sequence $\left\{n_{k}\right\} \subseteq \mathbb{N}$ and a map $S: D \rightarrow D$ such that

(i) $T^{n_{k}} S^{n_{k}} \rightarrow I_{D}$,

(ii) $T^{n_{k}} \rightarrow 0$, and

(iii) $S^{n_{k}} \rightarrow 0$,

pointwise in $D$, then $T$ is hypercyclic. Moreover, if

$$
n_{k}=k,
$$

for all $k \in \mathbb{N}$, then $T$ is topologically mixing.

The topological mixing part in the above result is due to Costakis and Sambarino (see Theorem 1.1, [15]).

For our purposes it is convenient to use the following version of the Hypercyclicity Criterion (cf. Definition 1.1 in [7] and also see [8]):

Theorem 2.1. (The Hypercyclicity Criterion) Let $X$ be a Banach space, $D$ be a dense set in $X$, and let $T \in \mathcal{B}(X)$. Suppose that $\left\{n_{k}\right\} \subseteq \mathbb{N}$ is a strictly increasing sequence. If

(i) $T^{n_{k}} \rightarrow 0$ pointwise on $D$, and

(ii) for each $f \in D$ there exists a sequence $\left\{f_{k}\right\}_{k \geq 1} \subseteq X$ such that

$$
f_{k} \rightarrow 0 \quad \text { and } \quad T^{n_{k}} f_{k} \rightarrow f
$$

then $T$ is hypercyclic. Moreover, if

$$
n_{k}=k,
$$

for all $k \in \mathbb{N}$, then $T$ is topologically mixing.

We now proceed to a chaoticity criterion (see Bonilla and Grosse-Erdmann [12, page 386): Let $T$ be an operator on a Banach space $X, D$ be dense in $X$ and $S: D \rightarrow D$ be a map. If

(i) $\sum_{n \geq 0} T^{n} x$ and $\sum_{n \geq 0} S^{n} x$ are unconditionally convergent, and

(ii) $T S x=x$,

for all $x \in D$, then $T$ is chaotic on $X$.

The above conditions are also sufficient for another important notion in linear dynamics, called frequent hypercyclicity, initiated by F. Bayart and S. Grivaux [5]. We refer the reader to [6] and [12] for an introductory discussion of the topic.

Recall that a series $\sum_{n} u_{n}$ in a Banach space $X$ is said to be unconditionally convergent if $\sum_{n} u_{\sigma(n)}$ is convergent for all permutations $\sigma$ on $\mathbb{N}$. It is well known that the unconditional convergence of $\sum_{n} u_{n}$ is equivalent to the following: for $\epsilon>0$, there exists $N \in \mathbb{N}$ such that

$$
\left\|\sum_{n \in F} u_{n}\right\|<\epsilon
$$

for all finite subsets $F$ of $\{N, N+1, N+2, \cdots\}$ (see, for instance [6], page 138).

The following version of the Chaoticity Criterion will be useful in what follows. This is probably known to experts, though we cannot find an exact reference (however, see Remark 9.10 in [20] and page 386 in [12]). 
Theorem 2.2. (Chaoticity Criterion) Let $X$ be a Banach space, $T \in \mathcal{B}(X)$ and $D$ be a dense set. Then $T$ is chaotic if for each $x \in D$, there exists a sequence $\left\{u_{k}\right\}_{k \geq 0}$ in $X$ with $u_{0}=x$ such that

$$
\sum_{n \geq 0} T^{n} x \quad \text { and } \quad \sum_{n \geq 0} u_{n}
$$

are unconditionally convergent, and

$$
T^{n} u_{k}=u_{k-n}
$$

for all $k \geq n$.

Proof. Observe that if $x \in D$, then

$$
T^{n} x \rightarrow 0 \text { and } f_{n}:=u_{n} \rightarrow 0,
$$

as $n \rightarrow \infty$. Moreover,

$$
T^{n} f_{n}=T^{n} u_{n}=u_{n-n}=u_{0}=x,
$$

for all $n \geq 0$, and hence, in particular

$$
T^{n} f_{n} \rightarrow x,
$$

as $n \rightarrow \infty$. This shows that $T$ satisfies the Hypercyclicity Criterion with respect to the same dense set $D$ and the sequence $n_{k}=k$ for all $k \in \mathbb{N}$, and hence $T$ is hypercyclic. It remains to show that $T$ has a dense set of periodic points in $X$. Define

$$
x_{p}=\sum_{n \geq 1} T^{n p} x+x+\sum_{n \geq 1} u_{n p},
$$

for all $p \geq 1$. By the unconditional convergence of the given series we have that $x_{p} \rightarrow x$, as $p \rightarrow \infty$. On the other hand

$$
T^{p} x_{p}=\left(\sum_{n \geq 1} T^{(n+1) p} x\right)+T^{p} x+\left(\sum_{n \geq 1} T^{p} u_{n p}\right)
$$

together with the properties of $\left\{u_{n}\right\}$ gives

$$
T^{p}\left(x_{p}\right)=x_{p},
$$

that is, $x_{p}$ is a periodic point for $T$. The result now follows from the fact that $D$ is dense in $X$ and $\left\{x_{p}\right\}$ approximates the element $x$ in $D$.

For our purposes (cf. Theorem 4.2) it is also relevant to recall the complete characterization of chaoticity for backward weighted shifts $B_{\lambda}$ on $l^{p}$ spaces (see Grosse-Erdmann [19]): $B_{\lambda}$ on $l^{p}$ is chaotic if and only if

$$
\sum_{n}\left(\lambda_{1} \lambda_{2} \cdots \lambda_{n}\right)^{-p}<\infty .
$$

The case $p=2$ yields that $M_{z}^{*}$ on $H^{2}(\beta)$ is chaotic if and only if

$$
\sum_{n} \beta_{n}^{2}<\infty \text {. }
$$




\section{Reproducing Kernel Hilbert spaces And DeRivatives}

We first recall the basics and constructions of reproducing kernel Hilbert spaces (see [3] and [25]). Let $\mathcal{E}$ be a Hilbert space. An operator-valued function $K: \mathbb{D} \times \mathbb{D} \rightarrow \mathcal{B}(\mathcal{E})$ is called an analytic kernel if $K$ is analytic in the first variable and

$$
\sum_{i, j=1}^{n}\left\langle K\left(z_{i}, z_{j}\right) \eta_{j}, \eta_{i}\right\rangle_{\mathcal{E}} \geq 0
$$

for all $\left\{z_{i}\right\}_{i=1}^{n} \subseteq \mathbb{D}$ and $\left\{\eta_{i}\right\}_{i=1}^{n} \subseteq \mathcal{E}$ and $n \in \mathbb{N}$. In this case there exists a Hilbert space $\mathcal{H}_{\mathcal{E}}(K)$ of $\mathcal{E}$-valued analytic functions on $\mathbb{D}$ such that

$$
\{K(\cdot, w) \eta: w \in \mathbb{D}, \eta \in \mathcal{E}\},
$$

is a total set in $\mathcal{H}_{\mathcal{E}}(K)$. Here for $w \in \mathbb{D}$ and $\eta \in \mathcal{E}$, the symbol $K(\cdot, w) \eta$ represents the function

$$
(K(\cdot, w) \eta)(z)=K(z, w) \eta
$$

for all $z \in \mathbb{D}$. It is easy to verify that

$$
\langle f, K(\cdot, w) \eta\rangle_{\mathcal{H}_{\mathcal{E}}(K)}=\langle f(w), \eta\rangle_{\mathcal{E}},
$$

for all $f \in \mathcal{H}_{\mathcal{E}}(K), w \in \mathbb{D}$ and $\eta \in \mathcal{E}$ (cf. [22]). In other words

$$
\left\langle e v_{w} f, \eta\right\rangle_{\mathcal{E}}=\langle f, K(\cdot, w) \eta\rangle_{\mathcal{H}_{\mathcal{E}}(K)},
$$

where $e v_{w}: \mathcal{H}_{\mathcal{E}}(K) \rightarrow \mathcal{E}$ is the (bounded) evaluation map defined by

$$
e v_{w} f=f(w)
$$

for all $w \in \mathbb{D}$ and $f \in \mathcal{H}_{\mathcal{E}}(K)$. We call the Hilbert space $\mathcal{H}_{\mathcal{E}}(K)$ the analytic reproducing kernel Hilbert space corresponding to the kernel $K$.

It also follows from (3.1) that the functions

$$
w \mapsto e v_{w} \in \mathcal{B}\left(\mathcal{H}_{\mathcal{E}}(K), \mathcal{E}\right),
$$

and

$$
w \mapsto K(z, w) \in \mathcal{B}(\mathcal{E}) \quad(z \in \mathbb{D}),
$$

are analytic and co-analytic on $\mathbb{D}$, respectively, and

$$
K(z, w)^{*}=K(w, z),
$$

and

$$
e v_{z} \circ e v_{w}^{*}=K(z, w)
$$

for all $z, w \in \mathbb{D}$. Furthermore, note that

$$
\begin{aligned}
\|K(\cdot, w) \eta\|_{\mathcal{H}_{\mathcal{E}}(K)}^{2} & =\langle K(\cdot, w) \eta, K(\cdot, w) \eta\rangle_{\mathcal{H}_{\mathcal{E}}(K)} \\
& =\langle K(w, w) \eta, \eta\rangle_{\mathcal{E}},
\end{aligned}
$$

that is

$$
\|K(\cdot, w) \eta\|_{\mathcal{H}_{\mathcal{E}}(K)}=\left\|K(w, w)^{1 / 2} \eta\right\|_{\mathcal{E}}
$$

for all $w \in \mathbb{D}$ and $\eta \in \mathcal{E}$.

Conversely, let $\mathcal{H}$ be a Hilbert space of analytic functions on $\mathbb{D}$ taking values in $\mathcal{E}$, and let the evaluation map $e v_{w}: \mathcal{H} \rightarrow \mathcal{E}$ (defined as above) be continuous for all $w \in \mathbb{D}$. Then $\mathcal{H}$ is an analytic reproducing kernel Hilbert space corresponding to the $\mathcal{B}(\mathcal{E})$-valued analytic kernel $K$ on $\mathbb{D}$ where $K(z, w)=e v_{z} \circ e v_{w}^{*}$ for all $z, w \in \mathbb{D}$. 
Now let $\mathcal{H}_{\mathcal{E}}(K)$ be an analytic reproducing kernel Hilbert space. Note that the $\mathcal{B}\left(\mathcal{H}_{\mathcal{E}}(K), \mathcal{E}\right)$ valued function $z \mapsto e v_{z}$ is analytic on $\mathbb{D}$. Indeed, for fixed $f \in \mathcal{H}_{\mathcal{E}}(K)$ and $\eta \in \mathcal{E}$, the function

$$
z \mapsto\left\langle e v_{z}(f), \eta\right\rangle_{\mathcal{E}}=\langle f(z), \eta\rangle_{\mathcal{E}}
$$

is analytic on $\mathbb{D}$. Since analyticity in the strong operator topology is equivalent to the analyticity in operator norm (cf. Chapter 5, Theorem 1.2, [29]), it follows that $z \mapsto e v_{z}$ is analytic on $\mathbb{D}$. Now if $z_{0} \in \mathbb{D}$, then we have the following power series (in powers of $\left.\left(z-z_{0}\right)\right)$ expansion

$$
e v_{z}=\left.\sum_{n \geq 0}\left(z-z_{0}\right)^{n} \frac{\partial^{n} e v_{z}}{\partial z^{n}}\right|_{z=z_{0}}
$$

in the operator norm topology. This implies that $\left.\frac{\partial^{n} e v_{z}}{\partial z^{n}}\right|_{z=z_{0}}: \mathcal{H}_{\mathcal{E}}(K) \rightarrow \mathcal{E}$ is a bounded linear operator for each $z_{0} \in \mathbb{D}$ and $n \geq 0$. Moreover, since

$$
\begin{aligned}
\left(\frac{e v_{z_{0}+h}-e v_{z_{0}}}{h}\right) f & =\frac{f\left(z_{0}+h\right)-f\left(z_{0}\right)}{h} \\
& \rightarrow f^{\prime}\left(z_{0}\right),
\end{aligned}
$$

as $h \rightarrow 0$, we have that

$$
\left.\frac{\partial e v_{z}}{\partial z}\right|_{z=z_{0}}(f)=f^{\prime}\left(z_{0}\right)
$$

for all $f \in \mathcal{H}_{\mathcal{E}}(K)$ and $z_{0} \in \mathbb{D}$. Similarly

$$
\left.\frac{\partial^{n} e v_{z}}{\partial z^{n}}\right|_{z=z_{0}}(f)=f^{(n)}\left(z_{0}\right)
$$

for all $f \in \mathcal{H}_{\mathcal{E}}(K)$ and $z_{0} \in \mathbb{D}$. On the other hand, the same argument as above can now be used to show that $\left.\frac{\partial^{n} e v_{w}^{*}}{\partial \bar{w}^{n}}\right|_{w=w_{0}}: \mathcal{E} \rightarrow \mathcal{H}_{\mathcal{E}}(K)$ is a bounded linear operator for each $w_{0} \in \mathbb{D}$ and $n \geq 0$. Now using the identity

$$
e v_{\lambda}^{*} \eta=K(\cdot, \lambda) \eta
$$

for all $\lambda \in \mathbb{D}$ and $\eta \in \mathcal{E}$, we find, for each $w_{0} \in \mathbb{D}$, that

$$
\begin{aligned}
\left(\frac{e v_{w_{0}+h}^{*}-e v_{w_{0}}^{*}}{\bar{h}}\right) \eta & =\frac{K\left(\cdot, w_{0}+h\right) \eta-K\left(\cdot, w_{0}\right) \eta}{\bar{h}} \\
& \rightarrow\left(\frac{\partial K}{\partial \bar{w}}\left(\cdot, w_{0}\right)\right) \eta
\end{aligned}
$$

as $h \rightarrow 0$. Thus

$$
\left.\frac{\partial e v_{w}^{*}}{\partial \bar{w}}\right|_{w=w_{0}}(\eta)=\left(\frac{\partial K}{\partial \bar{w}}\left(\cdot, w_{0}\right)\right) \eta
$$

and, again, we have that

$$
\left.\frac{\partial^{n} e v_{w}^{*}}{\partial \bar{w}^{n}}\right|_{w=w_{0}}(\eta)=\left(\frac{\partial^{n} K}{\partial \bar{w}^{n}}\left(\cdot, w_{0}\right)\right) \eta
$$


for all $w_{0} \in \mathbb{D}, \eta \in \mathcal{E}$ and $n \geq 0$. Moreover, we have

$$
\begin{aligned}
\left.\frac{\partial K(\cdot, w)}{\partial \bar{w}}\right|_{w=w_{0}}(\eta) & =\lim _{h \rightarrow 0} \frac{e v_{w_{0}+h}^{*}(\eta)-e v_{w_{0}}^{*}(\eta)}{\bar{h}} \\
& =\left(\left.\frac{\partial e v_{z}}{\partial z}\right|_{z=w_{0}}\right)^{*} \eta
\end{aligned}
$$

and similarly,

$$
\left.\frac{\partial^{n} K(\cdot, w)}{\partial \bar{w}^{n}}\right|_{w=w_{0}}=\left.\frac{\partial^{n} e v_{z}}{\partial z^{n}}\right|_{z=w_{0}} ^{*},
$$

for all $n \in \mathbb{Z}_{+}$. Furthermore, for all $f \in \mathcal{H}_{\mathcal{E}}(K), n \in \mathbb{Z}_{+}, w \in \mathbb{D}$ and $\eta \in \mathcal{E}$, it follows that

$$
\begin{aligned}
\left\langle f,\left(\left.\frac{\partial^{n} K}{\partial \bar{w}^{n}}(\cdot, w)\right|_{w=w_{0}}\right) \eta\right\rangle_{\mathcal{H}_{\mathcal{E}}(K)} & =\left.\frac{\partial^{n}}{\partial w^{n}}\left(\langle f, K(\cdot, w) \eta\rangle_{\mathcal{H}_{\mathcal{E}}(K)}\right)\right|_{w=w_{0}} \\
& =\left.\frac{\partial^{n}}{\partial w^{n}}\left(\langle f(w), \eta\rangle_{\mathcal{E}}\right)\right|_{w=w_{0}} \\
& =\left\langle f^{(n)}\left(w_{0}\right), \eta\right\rangle_{\mathcal{E}},
\end{aligned}
$$

that is

$$
\left\langle f^{(n)}\left(w_{0}\right), \eta\right\rangle_{\mathcal{E}}=\left\langle f,\left.\frac{\partial^{n} e v_{w}^{*}}{\partial \bar{w}^{n}}\right|_{w=w_{0}} \eta\right\rangle_{\mathcal{H}_{\mathcal{E}}(K)} .
$$

In particular, if $n \in \mathbb{Z}_{+}$and $\eta \in \mathcal{E}$, then the function

$$
z \mapsto\left(\frac{\partial^{n} K}{\partial \bar{w}^{n}}(z, 0)\right) \eta,
$$

is in $\mathcal{H}_{\mathcal{E}}(K)$, which follows from (3.3). Therefore, we have proved the following:

Theorem 3.1. Let $\mathcal{H}_{\mathcal{E}}(K)$ be an analytic reproducing kernel Hilbert space. Let

$$
K_{n}(z)=\frac{\partial^{n} K}{\partial \bar{w}^{n}}(z, 0),
$$

and let

$$
K_{n, \eta}(z)=K_{n}(z) \eta
$$

for all $z \in \mathbb{D}, \eta \in \mathcal{E}$ and $n \in \mathbb{Z}_{+}$. Then $K_{n}(z) \in \mathcal{B}(\mathcal{E})$ for all $z \in \mathbb{D}, K_{n, \eta} \in \mathcal{H}_{\mathcal{E}}(K)$ and

$$
\left\langle f^{(n)}(0), \eta\right\rangle_{\mathcal{E}}=\left\langle f, K_{n, \eta}\right\rangle_{\mathcal{H}_{\mathcal{E}}(K)},
$$

for all $f \in \mathcal{H}_{\mathcal{E}}(K), \eta \in \mathcal{E}$ and $n \in \mathbb{Z}_{+}$.

The following formulae for the inner product and norm are now immediate:

Corollary 3.2. In the setting of Theorem 3.1, we have

$$
\left\langle K_{m, \zeta}, K_{n, \eta}\right\rangle_{\mathcal{H}_{\mathcal{E}}(K)}=\left\langle\left(\frac{\partial^{n+m} K}{\partial z^{n} \partial \bar{w}^{m}}(0,0)\right) \zeta, \eta\right\rangle_{\mathcal{E}}
$$

for all $m, n \in \mathbb{Z}_{+}$and $\eta, \zeta \in \mathcal{E}$. In particular, we have

$$
\left\|K_{n, \eta}\right\|_{\mathcal{H}_{\mathcal{E}}(K)}^{2}=\left\langle\left(\frac{\partial^{2 n} K}{\partial z^{n} \partial \bar{w}^{n}}(0,0)\right) \eta, \eta\right\rangle_{\mathcal{E}} .
$$

Proof. By using the identity in Theorem 3.1 with $f=K_{m, \zeta}$, the result follows. 
For example, in the particular case of a scalar-valued kernel $K$, we have

$$
K(z, w)=\sum_{m, n \geq 0} a_{m n} z^{m} \bar{w}^{n}
$$

for some (not necessarily bounded) infinite matrix $\left(a_{m n}\right)$. Then the inner product and norm expressions in Corollary 3.2 are given by $n ! m ! a_{m n}$ and $(n !)^{2} a_{n n}$, respectively.

Theorem 3.1 and Corollary 3.2 should be compared with Lemmas 4.1 and 4.3 in [14] on generalized Bergman kernels.

Now let $M_{z}$ denote the multiplication operator on $\mathcal{H}_{\mathcal{E}}(K)$, that is

$$
\left(M_{z} f\right)(w)=w f(w)
$$

for all $f \in \mathcal{H}_{\mathcal{E}}(K)$ and $w \in \mathbb{D}$. If $M_{z}$ is bounded, $w \in \mathbb{D}, \eta \in \mathcal{E}$ and $f \in \mathcal{H}_{\mathcal{E}}(K)$, then

$$
\begin{aligned}
\left\langle M_{z}^{*}(K(\cdot, w) \eta), f\right\rangle_{\mathcal{H}_{\mathcal{E}}(K)} & =\langle K(\cdot, w) \eta, z f\rangle_{\mathcal{H}_{\mathcal{E}}(K)} \\
& =\langle\eta, w f(w)\rangle_{\mathcal{E}} \\
& =\langle\bar{w} \eta, f(w)\rangle_{\mathcal{E}} \\
& =\langle\bar{w} K(\cdot, w) \eta, f\rangle_{\mathcal{H}_{\mathcal{E}}(K)}
\end{aligned}
$$

that is

In particular

$$
M_{z}^{*}(K(\cdot, w) \eta)=\bar{w} K(\cdot, w) \eta
$$

$$
M_{z}^{*}(K(\cdot, 0) \eta)=0
$$

for all $\eta \in \mathcal{E}$. Now using

$$
K(\cdot, w) \eta=\sum_{n=0}^{\infty} \frac{1}{n !} K_{n, \eta} \bar{w}^{n}
$$

it follows from (3.4) that

$$
M_{z}^{*}\left(\sum_{n=0}^{\infty} \frac{1}{n !} K_{n, \eta} \bar{w}^{n}\right)=\bar{w} \sum_{n=0}^{\infty} \frac{1}{n !} K_{n, \eta} \bar{w}^{n},
$$

for all $w \in \mathbb{D}$ and $\eta \in \mathcal{E}$. Equating the coefficients of the same powers of $\bar{w}$ on either side, we immediately get the following "backward shift" property of $M_{z}^{*}$ :

Theorem 3.3. Let $\mathcal{H}_{\mathcal{E}}(K)$ be an analytic reproducing kernel Hilbert space. If the multiplication operator $M_{z}$ is bounded on $\mathcal{H}_{\mathcal{E}}(K)$, then

$$
M_{z}^{*}\left(\frac{1}{n !} K_{n, \eta}\right)= \begin{cases}\frac{1}{(n-1) !} K_{n-1, \eta} & \text { if } n \geq 1 \\ 0 & \text { if } n=0\end{cases}
$$

for all $\eta \in \mathcal{E}$.

\section{Dynamics of $M_{z}^{*}$}

In this section we present the main results on dynamics of $M_{z}^{*}$ on $\mathcal{H}_{\mathcal{E}}(K)$. Our first result concerns hypercyclicity and topological mixing. The second result is about the chaoticity of $M_{z}^{*}$.

We begin with the following definition: Let $I$ be a set, and let $\left\{u_{n}\right\}$ be a sequence of complex-valued functions on $I$. We say that $\liminf _{n} u_{n}(x)=0$ uniformly in $x \in I$, if 
there exists a subsequence $\left\{n_{k}\right\}$ of natural numbers such that $\lim _{k \rightarrow \infty} u_{n_{k}}(x)=0$ uniformly in $x \in I$.

Theorem 4.1. Let $\mathcal{E}$ be a Hilbert space, $\mathcal{E}_{0}$ a total subset in $\mathcal{E}$ and let $\mathcal{H}_{\mathcal{E}}(K)$ be an analytic reproducing kernel Hilbert space. If $M_{z}$ is bounded on $\mathcal{H}_{\mathcal{E}}(K)$, then the following hold:

(1) If

$$
\liminf _{n}\left(\frac{1}{(n !)^{2}}\left\langle\left(\frac{\partial^{2 n} K}{\partial z^{n} \partial \bar{w}^{n}}(0,0)\right) \eta, \eta\right\rangle_{\mathcal{E}}\right)=0,
$$

uniformly in $\eta \in \mathcal{E}_{0}$, then $M_{z}^{*}$ is hypercyclic.

(2) If

$$
\lim _{n}\left(\frac{1}{(n !)^{2}}\left\langle\left(\frac{\partial^{2 n} K}{\partial z^{n} \partial \bar{w}^{n}}(0,0)\right) \eta, \eta\right\rangle_{\mathcal{E}}\right)=0
$$

for all $\eta \in \mathcal{E}_{0}$, then $M_{z}^{*}$ is topologically mixing.

Proof. We apply Theorem 2 2.1, the Hypercyclicity Criterion, to $M_{z}^{*}$. For each $\eta \in \mathcal{E}_{0}$ and $m \in \mathbb{Z}_{+}$, define $\hat{K}_{m, \eta} \in \mathcal{H}_{\mathcal{E}}(K)$ (see Theorem 3.1) by

$$
\hat{K}_{m, \eta}(z)=\frac{1}{m !}\left(\frac{\partial^{m} K}{\partial \bar{w}^{m}}(z, 0)\right) \eta .
$$

Then the set $D$ is total in $\mathcal{H}_{\mathcal{E}}(K)$, where

$$
D=\operatorname{span}\left\{\hat{K}_{m, \eta}: m \in \mathbb{Z}_{+}, \eta \in \mathcal{E}_{0}\right\} .
$$

Indeed, if $g \in \mathcal{H}_{\mathcal{E}}(K)$ is orthogonal to $D$, then

$$
\left\langle g, \hat{K}_{m, \eta}\right\rangle=0
$$

and so, by Theorem 3.1, we have

$$
\left\langle g^{(m)}(0), \eta\right\rangle_{\mathcal{E}}=0
$$

for all $m \in \mathbb{Z}_{+}$and $\eta \in \mathcal{E}_{0}$. Since the span of $\mathcal{E}_{0}$ is dense in $\mathcal{E}$, it follows that

$$
g^{(m)}(0)=0,
$$

for all $m \in \mathbb{Z}_{+}$and hence

$$
g \equiv 0
$$

Now note that $\left\{\hat{K}_{m, \eta}: m \in \mathbb{Z}_{+}, \eta \in \mathcal{E}_{0}\right\}$ is a generating set for $D$. If $\zeta \in \mathcal{E}, s>t$ and $s, t \in \mathbb{Z}_{+}$, then by (3.5) and Theorem 3.3 , it follows that

$$
M_{z}^{* s} \hat{K}_{t, \zeta}=0 \text {. }
$$

Let

$$
f=\sum_{j=1}^{p} \alpha_{j} \hat{K}_{m_{j}, \eta_{j}} \in D,
$$

for some positive integer $p, \alpha_{j} \in \mathbb{C}, m_{j} \in \mathbb{Z}_{+}$and $\eta_{j} \in \mathcal{E}_{0}, 1 \leq j \leq p$. Then (4.1) implies, in particular, that

$$
M_{z}^{* n} f \rightarrow 0
$$

as $n \rightarrow \infty$. This proves the first condition of the criterion. 
To prove the second condition of the criterion, let

$$
f=\sum_{j=1}^{p} \alpha_{j} \hat{K}_{m_{j}, \eta_{j}} \in D,
$$

and first define a sequence $\left\{g_{n}\right\} \subseteq D$ by

$$
g_{n}:=\sum_{j=1}^{p} \alpha_{j} \hat{K}_{m_{j}+n, \eta_{j}},
$$

for all $n \in \mathbb{N}$. By Theorem 3.3 , it follows that

$$
M_{z}^{* n}\left(\hat{K}_{m_{j}+n, \eta_{j}}\right)=\hat{K}_{m_{j}, \eta_{j}},
$$

for $j=1, \ldots, p$, and hence

$$
M_{z}^{* n} g_{n}=f
$$

for $n \in \mathbb{N}$. Since

$$
\left\|\hat{K}_{m, \eta}\right\|_{\mathcal{H}_{\mathcal{E}}(K)}^{2}=\frac{1}{(m !)^{2}}\left\langle\left(\frac{\partial^{2 m} K}{\partial z^{m} \partial \bar{w}^{m}}(0,0)\right) \eta, \eta\right\rangle_{\mathcal{E}}
$$

for all $m \geq 0$, by Corollary 3.2 , and

$$
\liminf _{n}\left(\frac{1}{(n !)^{2}}\left\langle\left(\frac{\partial^{2 n} K}{\partial z^{n} \partial \bar{w}^{n}}(0,0)\right) \eta, \eta\right\rangle_{\mathcal{E}}\right)=0,
$$

uniformly in $\eta \in \mathcal{E}_{0}$ by assumption, it follows that there exists a sequence of natural numbers $\left\{t_{k}\right\}$ such that

$$
\hat{K}_{t_{k}, \eta} \rightarrow 0,
$$

in $\mathcal{H}_{\mathcal{E}}(K)$, for all $\eta \in \mathcal{E}_{0}$. This implies that

$$
M_{z}^{* j}\left(\hat{K}_{t_{k}, \eta}\right) \rightarrow 0,
$$

as $k \rightarrow \infty$ and for all $j \geq 0$. Now observe that

$$
\hat{K}_{t_{k}-j, \eta}=M_{z}^{* j}\left(\hat{K}_{t_{k}, \eta}\right) \text {, }
$$

for all $t_{k} \geq j$. Hence

$$
\hat{K}_{t_{k}-j, \eta} \rightarrow 0
$$

as $k \rightarrow \infty$ and for all $j \geq 0$.

We now use the following lemma (see Lemma 4.2, page 90, [20]): Let $(X, d)$ be a metric space and let $\left\{y_{k}\right\}$ be a sequence in $X$. Suppose that $\left\{t_{k}\right\}$ is a sequence of natural numbers. If the subsequence $\left\{y_{t_{k}-j}\right\}$ converges to a fixed element $y$ for each $j$, then there exists $\left\{n_{k}\right\}$ such that $\left\{y_{n_{k}+j}\right\}$ converges to $y$ for each $j$.

This shows that there exists a strictly increasing sequence of natural numbers $\left\{n_{k}\right\}_{k}$ such that

$$
\hat{K}_{n_{k}+j, \eta} \rightarrow 0
$$

for each $j$ and for all $\eta \in \mathcal{E}_{0}$. Set

that is

$$
f_{k}:=g_{n_{k}},
$$

$$
f_{k}=\sum_{j=1}^{p} \alpha_{j} \hat{K}_{n_{k}+m_{j}, \eta_{j}}
$$


for all $k \geq 1$. Clearly

$$
f_{k} \rightarrow 0,
$$

as $k \rightarrow \infty$. Moreover, by (4.2) we have

$$
M_{z}^{* n_{k}} f_{k}=f,
$$

for all $k \geq 1$. Hence $M_{z}^{*}$ satisfies the Hypercyclicity Criterion with respect to $\left\{n_{k}\right\}$. This proves (1).

For the second part, we proceed with $D$ and $f$ as in the proof of part (1) above. In this case, however, by assumption, it follows that

$$
\hat{K}_{n, \eta} \rightarrow 0,
$$

as $n \rightarrow \infty$ and for all $\eta \in \mathcal{E}_{0}$. If we set

$$
f_{n}=\sum_{j=1}^{p} \alpha_{j} \hat{K}_{n+m_{j}, \eta_{j}}
$$

for all $n \geq 1$, then, as in the proof of part (1), it follows that

$$
f_{n} \rightarrow 0 \text {, }
$$

as $n \rightarrow \infty$, and

$$
M_{z}^{* n} f_{n}=f,
$$

for all $n \geq 1$. This concludes the proof.

We now proceed to the chaos of $M_{z}^{*}$ on $\mathcal{H}_{\mathcal{E}}(K)$. A double series $\sum_{i, j \geq 0} u_{i, j}$ in a Hilbert space $\mathcal{H}$ is called $\mathcal{F}$-summable if for $\epsilon>0$ there exists $N \in \mathbb{N}$ such that

$$
\left\|\sum_{i, j \in F} u_{i, j}\right\|<\epsilon
$$

for all finite sets $F \subset\{N, N+1, \cdots\}$.

Theorem 4.2. Let $\mathcal{E}$ be a Hilbert space, $\mathcal{E}_{0}$ a total subset in $\mathcal{E}$ and let $\mathcal{H}_{\mathcal{E}}(K)$ be an analytic reproducing kernel Hilbert space. If $M_{z}$ is bounded on $\mathcal{H}_{\mathcal{E}}(K)$ and the double series

$$
\sum_{n, m \geq 0} \frac{1}{n ! m !}\left\langle\left(\frac{\partial^{n+m} K}{\partial z^{n} \partial \bar{w}^{m}}(0,0)\right) \eta, \eta\right\rangle_{\mathcal{E}}
$$

is $\mathcal{F}$-summable for all $\eta \in \mathcal{E}_{0}$, then $M_{z}^{*}$ chaotic.

Proof. Here we apply Theorem 2.2 , the Chaoticity Criterion, and proceed with the set $D$ as defined in the proof of Theorem 4.1, Assume that

$$
f:=\sum_{j=1}^{p} \alpha_{j} \hat{K}_{m_{j}, \eta_{j}} \in D
$$

and choose

$$
u_{k}:=\sum_{j=1}^{p} \alpha_{j} \hat{K}_{k+m_{j}, \eta_{j}} \quad(k \geq 0) .
$$


From the proof of Theorem 4.1 it follows that for $k \geq n$,

$$
M_{z}^{* n} u_{k}=\sum_{j=1}^{p} \alpha_{j} \hat{K}_{k-n+m_{j}, \eta_{j}}=u_{k-n} .
$$

Now using (4.1) we obtain that the series

$$
\sum_{n \geq 0} M_{z}^{* n} \hat{K}_{m, \eta}
$$

is unconditionally convergent in $\mathcal{H}_{\mathcal{E}}(K)$ for each $m \in \mathbb{Z}_{+}$and $\eta \in \mathcal{E}_{0}$, and so

$$
\sum_{n \geq 0} M_{z}^{* n} f
$$

is also unconditionally convergent in $\mathcal{H}_{\mathcal{E}}(K)$ for all $f \in D$. Now, we verify that the series $\sum_{n \geq 0} u_{n}$ is unconditionally convergent. It is, however, enough to check the above for

$$
f=\hat{K}_{m, \eta},
$$

where $m \in \mathbb{Z}_{+}$and $\eta \in \mathcal{E}_{0}$, that is, we need to verify that the series

$$
\sum_{n \geq 0} \hat{K}_{m+n, \eta}
$$

is unconditionally convergent in $\mathcal{H}_{\mathcal{E}}(K)$. This is equivalent to showing that

$$
\sum_{n \geq 0} \hat{K}_{n, \eta}
$$

is unconditionally convergent for all $\eta \in \mathcal{E}_{0}$. Indeed, for $\epsilon>0$ and $\eta \in \mathcal{E}_{0}$, by hypothesis, there exists $N \in \mathbb{N}$ such that

$$
\sum_{n, m \in F} \frac{1}{n ! m !}\left\langle\left(\frac{\partial^{n+m} K}{\partial z^{n} \partial \bar{w}^{m}}(0,0)\right) \eta, \eta\right\rangle_{\mathcal{E}}<\epsilon
$$

for all finite sets $F \subset[N, \infty) \cap \mathbb{N}$. Then Corollary 3.2 implies that

$$
\left\langle\sum_{n \in F} \hat{K}_{n, \eta}, \sum_{m \in F} \hat{K}_{m, \eta}\right\rangle<\epsilon
$$

that is

$$
\left\|\sum_{n \in F} \hat{K}_{n, \eta}\right\|_{\mathcal{H}_{\mathcal{E}}(K)}<\sqrt{\epsilon}
$$

Hence the series $\sum_{n>0} \hat{K}_{n, \eta}$ is unconditionally convergent in $\mathcal{H}_{\mathcal{E}}(K)$ (see the discussion preceding Theorem [2.2). This completes the proof of the theorem.

Of particular interest is the case where $K$ is a scalar-valued kernel. Here the sufficient condition for dynamics of $M_{z}^{*}$ involves the diagonal of the kernel function. We will address this issue in Theorem 5.1.

The converse of Theorem 4.1 is not true in general (see the example in Subsection 5.3 and also Section [6). 


\section{ExAmples AND APPliCATiOnS}

In this section we give concrete examples and applications of our main results.

5.1. Weighted shifts. We begin by noting that the weighted shift space $H^{2}(\beta)$, as introduced in Section 2, is an analytic reproducing kernel Hilbert space. In this case, the scalar-valued reproducing kernel function is given by

$$
K(z, w)=\sum_{n \geq 0} \beta_{n}^{2} z^{n} \bar{w}^{n}
$$

for all $z, w \in \mathbb{D}$. Moreover

$$
\frac{\partial^{n+m} K}{\partial z^{n} \bar{w}^{m}}(0,0)= \begin{cases}(n !)^{2} \beta_{n}^{2} & \text { if } m=n \\ 0 & \text { if } m \neq n\end{cases}
$$

and $m, n \geq 0$. By Theorem 4.1, it follows that $M_{z}^{*}$ is hypercyclic on $H^{2}(\beta)$ if

$$
\liminf _{n} \beta_{n}=0 .
$$

This condition is also necessary for hypercyclicity of $M_{z}^{*}$ on $H^{2}(\beta)$ (see Salas [27]) but not in general on $\mathcal{H}_{\mathcal{E}}(K)$ (see the Subsection 5.3). A similar classification result also holds for chaoticity and mixing for $M_{z}^{*}$ on $H^{2}(\beta)$ (cf. [20]).

5.2. Quasi-scalar reproducing kernel Hilbert spaces. We say that a kernel function $K: \mathbb{D} \times \mathbb{D} \rightarrow \mathcal{B}(\mathcal{E})$ is a quasi-scalar kernel if there exists a scalar-valued analytic kernel $k: \mathbb{D} \times \mathbb{D} \rightarrow \mathbb{C}$ such that

$$
K(z, w)=k(z, w) I_{\mathcal{E}}
$$

for all $z, w \in \mathbb{D}$. In this case, the general construction of reproducing kernel Hilbert spaces yields that (for instance, see [22])

$$
\mathcal{H}_{\mathcal{E}}(K) \cong \mathcal{H}(k) \otimes \mathcal{E}
$$

where $\mathcal{H}(k)$ is the reproducing kernel Hilbert space corresponding to the kernel function $k$ on $\mathbb{D}$. Quasi-scalar reproducing kernel Hilbert spaces play important roles in function theory and operator theory, particularly in the study of dilation theory and analytic model theory (cf. [22]).

As a simple example of the use of Theorems 4.1 and 4.2 , we prove the following:

Theorem 5.1. If $\mathcal{H}_{K}(\mathcal{E})$ is a quasi-scalar reproducing kernel Hilbert space and $M_{z}$ on $\mathcal{H}_{K}(\mathcal{E})$ is bounded, then:

(1) $M_{z}^{*}$ is hypercyclic if

$$
\liminf _{n} \frac{1}{(n !)^{2}} \frac{\partial^{2 n} k}{\partial z^{n} \partial \bar{w}^{n}}(0,0)=0 .
$$

(2) $M_{z}^{*}$ is topologically mixing if

$$
\lim _{n} \frac{1}{(n !)^{2}} \frac{\partial^{2 n} k}{\partial z^{n} \partial \bar{w}^{n}}(0,0)=0 .
$$

(3) $M_{z}^{*}$ is chaotic if the series

$$
\sum_{n, m \geq 0} \frac{1}{n ! m !} \frac{\partial^{n+m} k}{\partial z^{n} \partial \bar{w}^{m}}(0,0)
$$


is $\mathcal{F}$-summable.

Proof. If $k: \mathbb{D} \times \mathbb{D} \rightarrow \mathbb{C}$ is an analytic kernel on $\mathbb{D}$ and

$$
K(z, w)=k(z, w) I_{\mathcal{E}}
$$

for all $z, w \in \mathbb{D}$, then

$$
\|K(\cdot, w) \eta\|_{\mathcal{H}_{\mathcal{E}}(K)}=\|k(\cdot, w)\|_{\mathcal{H}(k)}\|\eta\|_{\mathcal{E}},
$$

for all $\eta \in \mathcal{E}$. Observe now that

$$
\left\langle\left(\frac{\partial^{n+m} K}{\partial z^{n} \partial \bar{w}^{m}}(0,0)\right) \eta, \eta\right\rangle_{\mathcal{E}}=\left(\frac{\partial^{n+m} k}{\partial z^{n} \partial \bar{w}^{m}}(0,0)\right)\|\eta\|_{\mathcal{E}}^{2}
$$

for all $w \in \mathbb{D}, \eta \in \mathcal{E}$ and $n, m \geq 0$. The result now follows from Theorems 4.1 and 4.2 ,

It is worth pointing out that the conclusion of the above theorem is independent of the choice of the Hilbert space $\mathcal{E}$. More specifically, if $k$ is a scalar kernel and

$$
\liminf _{n} \frac{1}{(n !)^{2}} \frac{\partial^{2 n} k}{\partial z^{n} \partial \bar{w}^{n}}(0,0)=0
$$

then $M_{z}^{*}$ on $\mathcal{H}_{\mathcal{E}}(K)$ is hypercyclic where

$$
K(z, w)=k(z, w) I_{\mathcal{E}}
$$

for all $z, w \in \mathbb{D}$ and $\mathcal{E}$ is a Hilbert space. This observation also should be compared with the linear dynamics of tensor products of operators (cf. [11] and [24]).

As a concrete application, consider the $\mathcal{E}$-valued Dirichlet space $\mathcal{D}_{\mathcal{E}}$ on $\mathbb{D}$, where $\mathcal{E}$ is separable Hilbert space. Notice that the kernel function for $\mathcal{D}_{\mathcal{E}}$ is given by

$$
(z, w) \mapsto\left(\sum_{n \geq 0} \frac{z^{n} \bar{w}^{n}}{n+1}\right) I_{\mathcal{E}} .
$$

From the above theorem, it then follows that $M_{z}^{*}$ is mixing on $\mathcal{D}_{\mathcal{E}}$.

5.3. A counter-example. Here we present a counterexample to show that the sufficient condition in Theorem 5.1 for $M_{z}^{*}$ to be hypercyclic is not a necessary condition.

Consider the Hilbert space $H^{2}(\beta) \subseteq \mathcal{O}(\mathbb{D})$, as in Section 2, corresponding to the (diagonal) kernel

$$
k(z, w)=\sum_{n \geq 0} \beta_{n}^{2} z^{n} \bar{w}^{n} \quad(z, w \in \mathbb{D}),
$$

where $\left\{\beta_{n}\right\}$ is a sequence of positive real numbers and

$$
\limsup _{n} \frac{\beta_{n+1}}{\beta_{n}} \leq 1
$$

Suppose that $M_{z, k}$, the multiplication operator by the coordinate function $z$, on $H^{2}(\beta)$ is bounded. Let

for all $z \in \mathbb{D}$, and set

$$
\theta(z)=\frac{1}{1-z}
$$

$$
\mathcal{H}=\left\{\theta f: f \in H^{2}(\beta)\right\} .
$$

Then $\mathcal{H}$ is a Hilbert space with the inner product

$$
\langle\theta f, \theta g\rangle_{\mathcal{H}}:=\langle f, g\rangle_{H^{2}(\beta)},
$$


for all $f, g \in H^{2}(\beta)$. Moreover, $\mathcal{H}$ is an analytic reproducing kernel Hilbert space corresponding to the kernel

$$
k_{\theta}(z, w)=\theta(z)\left(\sum_{n \geq 0} \beta_{n}{ }^{2} z^{n} \bar{w}^{n}\right) \overline{\theta(w)}
$$

for all $z, w \in \mathbb{D}$. Here the reproducing property is given by

$$
\left\langle\theta f, k_{\theta}(\cdot, w)\right\rangle_{\mathcal{H}}=\langle\theta f, \overline{\theta(w)} \theta k(\cdot, w)\rangle_{\mathcal{H}}=\theta(w)\langle f, k(\cdot, w)\rangle_{H^{2}(\beta)}=\theta(w) f(w),
$$

for all $f \in H^{2}(\beta)$ and $w \in \mathbb{D}$. Since

$$
\left\{\beta_{n} z^{n}\right\}_{n \geq 0}
$$

is an orthonormal basis in $H^{2}(\beta)$, it follows that

$$
\left\{\beta_{n} \theta z^{n}\right\}_{n \geq 0},
$$

forms an orthonormal basis in $\mathcal{H}$. Also observe that the multiplication operator $M_{z}$ on $\mathcal{H}$ is a bounded operator. Moreover, it follows that $M_{z}$ on $\mathcal{H}$ and $M_{z, k}$ on $H^{2}(\beta)$ are unitarily equivalent and hence so are $M_{z}^{*}$ and $M_{z, k}^{*}$. Since the condition

$$
\liminf _{n} \beta_{n}=0
$$

is necessary and sufficient for $M_{z, k}^{*}$ to be hypercyclic on $H^{2}(\beta)$ [27], under this assumption we conclude that $M_{z}^{*}$ is also hypercyclic on $\mathcal{H}$. However, the sufficient condition for hypercyclicity in Theorem 5.1 (and hence, that of Theorem 4.1) is not satisfied for $M_{z}^{*}$ on $\mathcal{H}$. Indeed, observe that

$$
k_{\theta}(z, w)=\left(1+z+z^{2}+\cdots\right)\left(\beta_{0}^{2}+\beta_{1}^{2} z \bar{w}+\beta_{2}^{2} z^{2} \bar{w}^{2}+\cdots\right)\left(1+\bar{w}+\bar{w}^{2}+\cdots\right),
$$

for all $z, w \in \mathbb{D}$. Then $a_{n n}$, the coefficient of $z^{n} \bar{w}^{n}$ in the above expansion, is given by

$$
a_{n n}=\beta_{0}^{2}+\beta_{1}^{2}+\cdots+\beta_{n}^{2}
$$

for all $n \geq 0$, and so

$$
\liminf _{n} a_{n n} \neq 0 .
$$

On the other hand

$$
a_{n n}=\frac{1}{(n !)^{2}} \frac{\partial^{2 n} k_{\theta}}{\partial z^{n} \partial \bar{w}^{n}}(0,0)
$$

for all $n \geq 0$. This shows that the sufficient condition for hypercyclicity in Theorem 4.1 is not necessary.

The construction above also allows us to work with non-zero polynomials instead of $\theta$. However, in this case, the sufficient condition in Theorem 5.1 for $M_{z}^{*}$ to be hypercyclic is also a necessary condition (see Section 6). 


\section{NeCESSARY CONDitions AND CONCluding REMARKS}

In the setting of analytic reproducing kernel Hilbert spaces many fundamental and basic questions about dynamics remain unanswered. For instance, the converse of Theorem 4.1 is false in general (see the example in Subsection 5.3). However, we can prove the converse for analytic tridiagonal reproducing kernel Hilbert spaces.

Choose two sequences of non-zero complex numbers $\mu=\left\{\mu_{n}\right\}$ and $\nu=\left\{\nu_{n}\right\}$ such that $\left\{e_{n}\right\}_{n \geq 0}$ is an orthonormal basis of a reproducing kernel Hilbert space (of analytic functions on $\mathbb{D}) \mathcal{H}_{\mu, \nu}$ (see Theorem 1 in [2]), where

$$
e_{n}(z)=\mu_{n} z^{n}+\nu_{n} z^{n+1} \quad(z \in \mathbb{D}, n \geq 0) .
$$

Since $k_{\mu, \nu}(z, w)=\sum_{n \geq 0} e_{n}(z) \overline{e_{n}(w)}$, we get the tridiagonal kernel as (cf. [2])

$$
k_{\mu, \nu}(z, w)=\left|\mu_{0}\right|^{2}+\sum_{n \geq 1}\left(\left|\mu_{n}\right|^{2}+\left|\nu_{n-1}\right|^{2}\right) z^{n} \bar{w}^{n}+\sum_{n \geq 0} \mu_{n} \overline{\nu_{n}} z^{n} \bar{w}^{n+1}+\sum_{n \geq 0} \overline{\mu_{n}} \nu_{n} z^{n+1} \bar{w}^{n}
$$

for all $z, w \in \mathbb{D}$. The analytic function Hilbert space $\mathcal{H}_{\mu, \nu}$ is called tridiagonal space (see Adams and McGuire [2] and Adams, McGuire and Paulsen [1]).

Further, suppose that

$$
\sup _{n \geq 0}\left|\mu_{n} / \mu_{n+1}\right|<\infty, \sup _{n \geq 0}\left|\nu_{n} / \mu_{n+1}\right|<1 .
$$

Then Theorem 5 in [2] ensures that $M_{z}$ is bounded on $\mathcal{H}_{\mu, \nu}$. Note also that, as pointed out in Theorem 4 in [2], forward weighted shifts are not unitarily equivalent to $M_{z}$ on $\mathcal{H}_{\mu, \nu}$, in general.

Now let $M_{z}^{*}$ be hypercyclic, and let $f:=k(\cdot, 0)$. Notice that

$$
f(z)=a+b z,
$$

where $a=\left|\mu_{0}\right|^{2}$ and $b=\mu_{0} \overline{\nu_{0}}$. Fix $n \geq 0$ and set

$$
z^{n} f=\sum_{j \geq 0} \alpha_{j} e_{j}
$$

that is

$$
a z^{n}+b z^{n+1}=\sum_{j \geq 0} \alpha_{j}\left(\mu_{j} z^{j}+\nu_{j} z^{j+1}\right),
$$

for some $\alpha_{j} \in \mathbb{C}$. Comparing coefficients of like powers, we have

$$
\alpha_{j}=0, \quad \alpha_{n}=\frac{a}{\mu_{n}}, \quad \alpha_{n+1}=\frac{b}{\mu_{n+1}}-\frac{a}{\mu_{n}} \frac{\nu_{n}}{\mu_{n+1}},
$$

for all $j<n$, and

$$
\alpha_{n+1+k}=-\frac{\nu_{n+k}}{\mu_{n+1+k}} \alpha_{n+k},
$$

for all $k \geq 1$. Set $M=\max \{a,|b|\}$. Since $R:=\sup _{m} \frac{\left|\nu_{m}\right|}{\left|\mu_{m+1}\right|}<1$, it follows that

$$
\left|\alpha_{n+1}\right|^{2} \leq\left(\frac{1}{\left|\mu_{n+1}\right|}+\frac{1}{\left|\mu_{n}\right|}\right)^{2} M^{2}
$$


and so

$$
\left|\alpha_{n+1+k}\right|^{2} \leq\left(\frac{1}{\left|\mu_{n+1}\right|}+\frac{1}{\left|\mu_{n}\right|}\right)^{2} M^{2} R^{2 k}
$$

for all $k \geq 1$. Since

$$
\left\|z^{n} f\right\|_{\mathcal{H}_{\mu, \nu}}^{2}=\sum_{j \geq 0}\left|\alpha_{j}\right|^{2}
$$

we have

$$
\begin{aligned}
\left\|z^{n} f\right\|_{\mathcal{H}_{\mu, \nu}}^{2} \leq & M^{2}\left(\frac{1}{\left|\mu_{n}\right|^{2}}+\left(\frac{1}{\left|\mu_{n+1}\right|}+\frac{1}{\left|\mu_{n}\right|}\right)^{2}+\left(\frac{1}{\left|\mu_{n+1}\right|}+\frac{1}{\left|\mu_{n}\right|}\right)^{2} R^{2}\right. \\
& \left.+\left(\frac{1}{\left|\mu_{n+1}\right|}+\frac{1}{\left|\mu_{n}\right|}\right)^{2} R^{4}+\cdots\right) \\
\leq & M^{2}\left(\frac{1}{\left|\mu_{n}\right|^{2}}+\frac{(r+1)^{2}}{\left|\mu_{n}\right|^{2}} \sum_{k \geq 1} R^{2 k}\right) \\
\leq & C \frac{1}{\left|\mu_{n}\right|^{2}}
\end{aligned}
$$

where $r=\sup _{n}\left(\left|\mu_{n}\right| /\left|\mu_{n+1}\right|\right)$ and $C=M^{2}\left(1+(r+1)^{2} \sum_{k} R^{2 k}\right)$. Now, if possible, let

$$
\liminf _{n}\left|\mu_{n}\right|>0 \text {. }
$$

Then the sequence $\left\{\left\|z^{n} f\right\|_{\mathcal{H}_{\mu, \nu}}\right\}_{n \geq 0}$ is bounded. Now let $g$ be a non-zero vector in $\mathcal{H}_{\mu, \nu}$. Since

$$
\left\langle M_{z}^{* n} g, f\right\rangle_{\mathcal{H}_{\mu, \nu}}=\left\langle g, z^{n} f\right\rangle_{\mathcal{H}_{\mu, \nu}}
$$

we deduce that $\left\{M_{z}^{* n} g\right\}_{n \geq 0}$ is a bounded set. This contradicts the fact that $M_{z}^{*}$ has a hypercyclic vector, and hence

$$
\liminf _{n}\left|\mu_{n}\right|=0
$$

Finally, since

$$
\frac{1}{(n !)^{2}} \frac{\partial^{2 n} k_{\mu, \nu}}{\partial z^{n} \partial \bar{w}^{n}}(0,0)=\left|\mu_{n}\right|^{2}+\left|\nu_{n-1}\right|^{2}=\left|\mu_{n}\right|^{2}\left(1+\left|\nu_{n-1}^{2} / \mu_{n}^{2}\right|\right) \leq 2\left|\mu_{n}\right|^{2},
$$

for all $n \geq 1$, it follows that

$$
\liminf _{n} \frac{1}{(n !)^{2}} \frac{\partial^{2 n} k_{\mu, \nu}}{\partial z^{n} \partial \bar{w}^{n}}(0,0)=0
$$

which is equivalent to (6.2).

A similar method applies also to the topological mixing property of $M_{z}^{*}$. Here we use the following fact: If $T$ is a mixing operator on a Banach space $X$, then $\lim _{n}\left\|T^{* n} x^{*}\right\|=\infty$ for all non-zero $x^{*} \in X^{*}$, where $X^{*}$ is the dual of $X$ (see Lemma 2.2(2), [10]). This together with the sufficient conditions in Theorem 5.1 yields the following complete characterization:

Theorem 6.1. Consider the space $\mathcal{H}_{\mu, \nu}$ defined as above, and assume that $\left\{\mu_{n}\right\}$ and $\left\{\nu_{n}\right\}$ satisfies the condition (6.1). Then 
(i) $M_{z}^{*}$ on $\mathcal{H}_{\mu, \nu}$ is hypercyclic if and only if

$$
\liminf _{n} \frac{1}{(n !)^{2}} \frac{\partial^{2 n} k_{\mu, \nu}}{\partial z^{n} \partial \bar{w}^{n}}(0,0)=0
$$

(ii) $M_{z}^{*}$ on $\mathcal{H}_{\mu, \nu}$ is topologically mixing if and only if

$$
\lim _{n} \frac{1}{(n !)^{2}} \frac{\partial^{2 n} k_{\mu, \nu}}{\partial z^{n} \partial \bar{w}^{n}}(0,0)=0 .
$$

We now confine our attention to the (counter-)examples in Subsection 5.3. Here we again obtain a complete characterization of dynamics by replacing the analytic function $\theta$ (defined by $\theta(z)=\frac{1}{1-z}$ ) by (operator-valued) analytic polynomials.

Let $\mathcal{E}$ be a Hilbert space and let $P(z)$ be a $\mathcal{B}(\mathcal{E})$-valued (analytic) polynomial. Suppose that

$$
P(z)=A_{0}+A_{1} z+\cdots+A_{d} z^{d}
$$

for some $A_{j} \in \mathcal{B}(\mathcal{E}), j=0, \ldots, d$. Now we consider a scalar-valued analytic (and diagonal) kernel $k$ on $\mathbb{D}$ :

$$
k(z, w)=\sum_{n \geq 0} \beta_{n}^{2} z^{n} \bar{w}^{n},
$$

where $\beta_{n}$ are non-negative numbers, and let $\mathcal{H}_{\mathcal{E}}(K)$ denote the reproducing kernel Hilbert space corresponding to the kernel

$$
K(z, w)=k(z, w) I_{\mathcal{E}}
$$

for all $z, w \in \mathbb{D}$. Let

$$
K_{P}(z, w)=P(z) K(z, w) P(w)^{*},
$$

for all $z, w \in \mathbb{D}$. Then $K_{P}$ is a $\mathcal{B}(\mathcal{E})$-valued kernel on $\mathbb{D}$. Set

$$
\mathcal{H}_{P}=\left\{P f: f \in \mathcal{H}_{\mathcal{E}}(K)\right\}
$$

where

$$
(P f)(z)=P(z) f(z)
$$

for all $f \in \mathcal{H}_{\mathcal{E}}(K)$ and $z \in \mathbb{D}$. Clearly $\mathcal{H}_{P}$ is a vector space of $\mathcal{E}$-valued analytic functions on $\mathbb{D}$. Now suppose that $A_{0}$ is injective. If $f=\sum_{n \geq 0} \eta_{n} z^{n} \in \mathcal{H}_{\mathcal{E}}(K)$ and

$$
P(z) f(z)=0
$$

for all $z \in \mathbb{D}$, then

$$
P(0) f(0)=A_{0} \eta_{0}=0,
$$

implies that $\eta_{0}=0$. Now differentiating (6.4), one gets

$$
P^{\prime}(z) f(z)+P(z) f^{\prime}(z)=0,
$$

and hence

$$
0=P^{\prime}(0) f(0)+P(0) f^{\prime}(0)=A_{0} \eta_{1} .
$$

We have $\eta_{1}=0$. Continuing in this way we obtain that $f \equiv 0$. It now follows that $\mathcal{H}$ is a Hilbert space with inner product defined by

$$
\langle P f, P g\rangle_{\mathcal{H}_{P}}:=\langle f, g\rangle_{\mathcal{H}_{\mathcal{E}}(K)},
$$

for all $f$ and $g$ in $\mathcal{H}_{\mathcal{E}}(K)$. 
Now we show that $\mathcal{H}_{P}$ is the reproducing kernel Hilbert space corresponding to the kernel $K_{P}$. Let $\left\{P f_{n}\right\} \subseteq \mathcal{H}_{P}$, and let $P f_{n} \rightarrow 0$ in $\mathcal{H}_{P}$ as $n \rightarrow \infty$. Since

$$
\left\|P f_{n}\right\|_{\mathcal{H}_{P}}=\left\|f_{n}\right\|_{\mathcal{H}_{\mathcal{E}}(K)}
$$

for all $n \geq 0$, it follows that $f_{n} \rightarrow 0$ as $n \rightarrow \infty$ in $\mathcal{H}_{\mathcal{E}}(K)$. Now, since $\mathcal{H}_{\mathcal{E}}(K)$ is a reproducing kernel Hilbert space, the evaluation operators are continuous. Therefore

$$
f_{n}(w) \rightarrow 0 \quad(w \in \mathbb{D}),
$$

in $\mathcal{E}$ as $n \rightarrow \infty$. Hence

$$
P(w) f_{n}(w) \rightarrow 0 \quad(w \in \mathbb{D}),
$$

in $\mathcal{E}$ as $n \rightarrow \infty$. This implies that the evaluation operators on $\mathcal{H}_{P}$ are bounded. Hence $\mathcal{H}_{P}$ is a reproducing kernel Hilbert space. Now, for any $\eta \in \mathcal{E}$ and $w \in \mathbb{D}$ we have that

$$
K_{P}(\cdot, w) \eta=P\left(k(\cdot, w) P(w)^{*} \eta\right) \in \mathcal{H}_{P},
$$

and

$$
\begin{aligned}
\left\langle P f, P\left(k(\cdot, w) P(w)^{*} \eta\right)\right\rangle_{\mathcal{H}_{P}} & =\left\langle f, k(\cdot, w) P(w)^{*} \eta\right\rangle_{\mathcal{H}_{\mathcal{E}}(K)} \\
& =\left\langle f(w), P(w)^{*} \eta\right\rangle_{\mathcal{E}} \\
& =\langle P(w) f(w), \eta\rangle_{\mathcal{E}}
\end{aligned}
$$

for all $f \in \mathcal{H}_{\mathcal{E}}(K)$. Hence $K_{P}$ is the kernel of $\mathcal{H}_{P}$.

Now, we observe that if $M_{z, K}$ (the multiplication operator on $\mathcal{H}_{\mathcal{E}}(K)$ by the coordinate function $z$ ) is bounded, then $M_{z, K}$ on $\mathcal{H}_{\mathcal{E}}(K)$ and $M_{z}$ on $\mathcal{H}_{P}$ are unitarily equivalent. Moreover, since $M_{z, K}$ on $\mathcal{H}_{\mathcal{E}}(K)$ and $M_{z, k} \otimes I$ on $\mathcal{H}(k) \otimes \mathcal{E}$ are unitarily equivalent, by a result of Martínez-Giménez and Peris (Proposition 1.14 in [24]) and Salas [27], it follows that $M_{z}^{*}$ is hypercyclic on $\mathcal{H}_{P}$ if and only if

$$
\liminf _{n} \beta_{n}=0 .
$$

Now equating the coefficients of $z^{n} \bar{w}^{n}$, say $C_{n n}$, in the expansion of $K_{P}(z, w)$ in (6.3), we have (as in Subsection 5.3)

$$
C_{n n}=A_{0} A_{0}^{*} \beta_{n}^{2}+A_{1} A_{1}^{*} \beta_{n-1}^{2}+\cdots+A_{d} A_{d}^{*} \beta_{n-d}^{2},
$$

for all $n \geq d$. Since $M_{z, k}$ is bounded, it follows that

$$
\sup _{k} \frac{\beta_{k}}{\beta_{k+1}}<\infty
$$

and hence, there exists $M>0$ such that

$$
\left\|C_{n n}\right\|_{\mathcal{B}(\mathcal{E})} \leq M \beta_{n}^{2}
$$

for all $n \geq 1$. Thus, if $M_{z}^{*}$ is hypercyclic on $\mathcal{H}_{P}$, then, by the above observation, we see that

$$
\liminf _{n}\left\|C_{n n}\right\|_{\mathcal{B}(\mathcal{E})}=0
$$

Hence, by

$$
C_{n n}=\frac{1}{(n !)^{2}} \frac{\partial^{2 n} K_{P}}{\partial z^{n} \partial \bar{w}^{n}}(0,0)
$$


for all $n \geq 0$, it follows that

$$
\liminf _{n}\left(\frac{1}{(n !)^{2}}\left\langle\left(\frac{\partial^{2 n} K_{P}}{\partial z^{n} \partial \bar{w}^{n}}(0,0)\right) \eta, \eta\right\rangle_{\mathcal{E}}\right)=0
$$

uniformly in $\eta \in \mathcal{E}_{1}$, where $\mathcal{E}_{1}$ is the open unit ball in $\mathcal{E}$. This proves the converse of the hypercyclicity part in Theorem 4.1. We have therefore shown the following result:

Theorem 6.2. Let $\mathcal{E}$ be a Hilbert space, $k$ on $\mathbb{D}$ be a scalar-valued kernel and

$$
k(z, w)=\sum_{n \geq 0} \beta_{n}^{2} z^{n} \bar{w}^{n},
$$

where $\beta_{n}$ are non-negative numbers, and

$$
\limsup _{n} \frac{\beta_{n+1}}{\beta_{n}} \leq 1
$$

Let $\mathcal{H}_{\mathcal{E}}(K)$ denote the reproducing kernel Hilbert space corresponding to the kernel

$$
K(z, w)=k(z, w) I_{\mathcal{E}}
$$

for all $z, w \in \mathbb{D}$. Suppose that $\left\{A_{j}\right\}_{j=0}^{d} \subseteq \mathcal{B}(\mathcal{E}), A_{0}$ is injective,

$$
P(z)=A_{0}+A_{1} z+\cdots+A_{d} z^{d},
$$

and let $\mathcal{H}_{P}$ denote the reproducing kernel Hilbert space corresponding to the kernel function

$$
K_{P}(z, w)=P(z) K(z, w) P(w)^{*} \quad(z, w \in \mathbb{D}) .
$$

If $M_{z}$ is bounded on $\mathcal{H}_{P}$, then the following are equivalent:

(i) $M_{z}^{*}$ is hypercyclic.

(ii) $\liminf _{n}\left(\frac{1}{(n !)^{2}}\left\langle\left(\frac{\partial^{2 n} K_{P}}{\partial z^{n} \partial \bar{w}^{n}}(0,0)\right) \eta, \eta\right\rangle_{\mathcal{E}}\right)=0$ uniformly in $\eta \in \mathcal{E}_{1}$, where $\mathcal{E}_{1}$ is the open unit ball in $\mathcal{E}$.

(iii) $\liminf \beta_{n}=0$.

The same method of proof also applies to the scalar-valued reproducing kernel Hilbert space $\mathcal{H}_{P}$ corresponding to the kernel function

$$
k_{P}(z, w)=P(z) k(z, w) \overline{P(w)} \quad(z, w \in \mathbb{D}),
$$

where

$$
P(z)=a_{0}+a_{1} z+\cdots+a_{d} z^{d},
$$

is a non-zero scalar polynomial and $k$ is defined as in Theorem 6.2 (observe that $k_{P}(z, w)$ is not a diagonal kernel in general). Here $\mathcal{H}_{P}=\{P f: f \in \mathcal{H}(k)\}$ and the inner product is given by $\langle P f, P g\rangle_{\mathcal{H}_{P}}=\langle f, g\rangle_{\mathcal{H}(k)}$ for all $f, g \in \mathcal{H}(k)$.

Theorem 6.3. If $M_{z}$ is bounded on $\mathcal{H}_{P}$, then the following are equivalent:

(i) $M_{z}^{*}$ is hypercyclic.

(ii) $\liminf _{n} \frac{1}{(n !)^{2}}\left(\frac{\partial^{2 n} k}{\partial z^{n} \partial \bar{w}^{n}}(0,0)\right)=0$.

(iii) $\liminf _{n} \beta_{n}=0$. 
It would be therefore very interesting to determine the class of vector valued analytic kernel functions for which the converse of Theorem 4.1 holds.

Acknowledgments. The authors would like to express their sincerest gratitude to the referee for careful reading of the manuscript, thoughtful remarks, and for many valuable suggestions. The authors are also grateful to Deepak Pradhan for a careful reading of the manuscript and valuable comments. The first author is grateful to the Indian Statistical Institute Bangalore for offering a visiting position. The first author is also supported in part by the NBHM post doctoral fellowship, File No. 0104/43/2017/RD-II/15354. The second author is supported in part by the Mathematical Research Impact Centric Support (MATRICS) grant, File No: MTR/2017/000522, by the Science and Engineering Research Board (SERB), Department of Science \& Technology (DST), Government of India, and NBHM (National Board of Higher Mathematics, DAE, India) Research Grant NBHM/R.P.64/2014.

\section{REFERENCES}

1. G. T. Adams, P. J. McGuire and V. Paulsen, Analytic reproducing kernels and multiplication operators, Illinois J. Math. 36 (1992), 404-419.

2. G. T. Adams and P. J. McGuire, Analytic tridiagonal reproducing kernels, J. London Math. Soc. (2) 64 (2001) 722-738.

3. N. Aronszajn, Theory of reproducing kernels, Trans. Amer. Math. Soc., 68 (1950) 337-404.

4. A. D. Baranov, Polynomials in the de Branges spaces of entire functions, Ark. Mat. 44 (2006) 16-38.

5. F. Bayart and S. Grivaux, Frequently hypercyclic operators, Trans. Amer. Math. Soc. 358 (2006) 5083-5117.

6. F. Bayart and E. Matheron, Dynamics of Linear Operators, Camb. Univ. Press. 179 (2009).

7. L. Bernal-Gonzalez, K.-G. Grosse-Erdmann, The hypercyclicity criterion for sequences of operators Studia Math. 157 (2003) 17-32.

8. J. Bes, A. Peris, Hereditarily hypercyclic operators J. Funct. Anal. 167 (1999) 94-112.

9. G. D. Birkhoff, Demonstration d'un theoreme elementaire sur les fonctions entires, C.R. Acad. Sci. Paris. 189 (1929).

10. J. Bonet, Dynamics of the differentiation operator on weighted spaces of entire functions, Math. Z. 261 (2009), 649-657.

11. J. Bonet, F. Martínez-Giménez and A. Peris, Universal and chaotic multipliers on spaces of operators, J. Math. Anal. Appl 297 (2004), 599-611.

12. A. Bonilla and K.-G. Grosse-Erdmann, Frequently hypercyclic operators and vectors, Ergodic Theory Dynamical Systems. 27 (2007), 383-404.

13. K. C. Chan and J. H. Shapiro, The cyclic behavior of translation operators on Hilbert spaces of entire functions, Indiana. Univ. Math. J. 40 (1991), 1421-1449.

14. R. Curto and N. Salinas, Generalized Bergman kernels and the Cowen-Douglas theory, Amer. J. Math. 106 (1984), 447-488.

15. G. Costakis and M. Sambarino, Topologically mixing hypercyclic operators, Proc. Amer. Math Soc. 132 (2004), 385-389.

16. A. G. Garcia, M. A. Hernandez-Medina, and A. Portal, Hypercyclicity of translation operators in a reproducing kernel Hilbert space of entire functions induced by an analytic Hilbert-space-valued kernel, Osaka J. Math. 52 (2015), 581-599.

17. R. M. Gethner and J. H. Shapiro, Universal vectors for operators on spaces of holomorphic functions, Proc Amer. Math. Soc. 100 (1987), 281-288.

18. G. Godefroy and J.H. Shapiro, Operators with dense, invariant, cyclic vector manifolds, J. Funct. Anal., 98 (1991), 229-269.

19. K.-G. Grosse-Erdmann, Hypercyclic and chaotic weighted shifts, Studia Math., 139 (2000), 47-68. 
20. K.-G. Grosse-Erdmann and A. Peris, Linear Chaos, Springer Universitext, 2011.

21. C. Kitai, Invariant closed sets for linear operators, Ph.D thesis, University of Toronto, Toronto (1982).

22. R. Kumari, J. Sarkar, S. Sarkar and D. Timotin Factorization of kernels and reproducing kernel Hilbert spaces, Integral Equations Operator Theory, 87 (2017), 225-244.

23. M. R. MacLane, Sequences of derivatives and normal families, J. Analyse. Math. 2 (1952/53), 72-87.

24. F. Martínez-Giménez and A. Peris, Universality and chaos for tensor produtcs of operators, J. Approx. Theory, 124 (2003), 7-24.

25. V. Paulsen and M. Raghupathi, An introduction to the theory of reproducing kernel Hilbert spaces, Cambridge Studies in Advanced Mathematics, 2016.

26. S. Rolewicz, On orbits of elements, Studia Math. 32 (1969), 17-22.

27. H. N. Salas, Hypercyclic weighted shifts, Trans. Amer. Math. Soc. 347 (1995) 1993-1004.

28. A.L. Shields, Weighted shift operators and analytic function theory, Topics of Operator Theory, Math. Surveys Monogr., vol 13, American Math. Soc. Providence, RI, 1974, 49-128.

29. A.E. Taylor and D.C. Lay, Introduction to Functional Analysis, 2nd edition, John Wiley \& Sons, New York, 1980.

Indian Statistical Institute, Statistics and Mathematics Unit, 8th Mile, Mysore Road, BANGALORE, 560059, INDIA

E-mail address: aneeshkolappa@gmail.com

Indian Statistical Institute, Statistics and Mathematics Unit, 8th Mile, Mysore Road, BANGALORE, 560059, INDIA

E-mail address: jay@isibang.ac.in, jaydeb@gmail.com 